\title{
A Composite Weight based Multiple Attribute Decision Support System for the Selection of Automated uided Vehicles
}

\author{
Vishram B. Sawant \\ Department of Mechanical Engineering, \\ Government College of Engineering, \\ Vidyanagar, Karad, 415124, Maharashtra, India.
}

\author{
Suhas S. Mohite \\ Department of Mechanical Engineering, \\ Government College of Engineering, \\ Vidyanagar, Karad, 415124, Maharashtra, India.
}

\begin{abstract}
This paper proposes a decision support system which integrates the objective weights of importance of the attributes as well as the subjective preferences of the decision maker to decide the composite weights of importance of the attributes. Using fuzzy set theory the qualitative attributes are converted into the quantitative attributes. Based on this model, a decision support system AGVSEL is developed for the selection of AGVs. AGVs are ranked by using the technique for order preference by similarity to ideal solution (TOPSIS), block TOPSIS and modified synthetic evaluation method (M-TOPSIS). The effectiveness of the support system is demonstrated with an illustrative example. The computational results obtained enable evaluation and selection of an appropriate AGV. Sensitivity analysis reveals that at a moderate value of interpolating factor rank transition takes place for topmost position thereby achieving better insight into the complex interplay of subjective and objective weights. Finally, the results of the proposed approach are compared with the results obtained by published methods. Thus, the proposed weight method with AGVSEL system improves decision making in MADM environment.
\end{abstract}

\section{Keywords:}

Multiple attribute decision making, automated guided vehicle, decision support system, TOPSISifx

\section{INTRODUCTION}

AGVs are battery-powered, unmanned vehicles with programming capabilities for path selection and positioning. They are capable of responding readily to frequently changing transport patterns and they can be integrated in fully automated manufacturing systems. These features make AGVs a viable alternative to other material handling methods. On the assumption that the labor costs will rise even more sharply in the future, AGVs will increase productivity, and improve system flexibility. The increasing interest in AGVs is reflected in the sales figures which reached a new peak in 2006 with a volume of \$281.7 million according to a yearly survey among European AGV producers carried out by the Department of Planning and Controlling of Warehouse and Transport Systems, Leibniz University Hannover, Germany [1]. AGV applications can be found throughout all industrial branches, from the automotive, printing, pharmaceutical sectors, metal, food processing to aerospace and port facilities.

An effective selection among more than 76 AGV manufacturers and different AGV models available in market requires the consideration of many quantitative and qualitative attributes. An MADM method is a procedure that specifies how attribute infor- mation is to be processed in order to arrive at a better choice. AGV selection attribute is defined as a factor that influences the selection of an AGV for a given application. These attributes include: costs involved, floor space requirements, load carrying capacity, travel speed, lift height, turning radius, travel patterns, programming flexibility, labor requirements, expandability, ease of operation, maintenance aspects, payback period, reconfiguration time, hardware targebility, support for modularity (i.e. ease of integrating with FMS, ASRS, and robots), robustness etc. AGV manufacturers develop AGVs of a variety of types and sizes. One company does not cover all niches equally well. Therefore, it is wise to evaluate AGVs based on a number of attributes. The decision making process in the selection of AGV consists of a combination of a variety of multidimensional attributes (see, Fig. 1). It includes: performance parameters, technical specifications, economical considerations and future expansion issues. One company does not cover all attributes equally well. Therefore, it is wise to evaluate AGVs based on a number of attributes.

To help address the issue of effective evaluation and selection of AGVs, various mathematical and systems modeling approaches have been proposed. The development of an intelligent material handling equipment selection system called material handling equipment selection advisor (MHESA) is described in [2]. In addition to above, [3] developed decision support system for the selection of material handling equipments (SEMH). SEMH searches its knowledge base to recommend the type of material handling equipment to be used based on various characteristics like type, weight, size, etc. FUMAHES-fuzzy multiattribute material handling equipment selection system consists of a database and a rule-based system [4]. In other application,[5] focused on the application of the AHP technique in selecting the optimal material handling equipment for a specific material handling equipment. AHP - PROMETHEE integrated approach for selection of the most suitable equipment used by [6]. A decision support system to help the decision makers in their machining center selection decisions was developed by [7]. One of the recent study proposed by [8], An integrated fuzzy multi-criteria decision making methodology for material handling equipment selection problem MHESP. The proposed methodology is based on fuzzy sets, Analytic Network Process (ANP) and Preference Ranking Organization METHod for Enrichment Evaluations (PROMETHEE) approaches. A new methodology for multi-attribute selection of automated guided vehicle (AGV) for the material handling. A modified grey relational analysis (M-GRA) method combined with AHP has been used for selection of AGVs [9].

There are several limitations of existing expert systems for material handling equipment selection. Most of them are incomplete prototypes that consider only a limited number of equipment types and attributes. However, there is a need for a simple, 
systematic and self-sufficient support system to guide the user organizations in taking a decision in selecting a AGV. To the best of our knowledge, there is no decision making system that has been developed specifically for AGV selection considering available AGVs in the market. So also, different decision makers might assign different weights to different attributes; however, none of these systems provide the means for the user to select their own weightings on basis of subjective and objective information integration. The proposed system in this paper, namely AGVSEL (Automated Guided Vehicle SELection), overcomes above limitations. It also uses crisp (non-fuzzy) and fuzzy MADM approaches which take into account the uncertainties and imprecision that may be associated with the decision making approach. The aim of the present paper is to propose a new MADM method where, composite weight is computed by integrating the objective and subjective weights obtained by Shannon's entropy method and Satty's analytic hierarchy process (AHP) with different versions of TOPSIS. Fuzzy set theory can be utilized to cover the vagueness inherent in the nature of this selection problem. Experts express their judgments on the value of each alternative with respect to defined attribute in linguistic terms. This helps the model to be more user friendly and realistic. The remainder of this paper is organized as follows: Section 2 describes the composite weight MADM method. Section 3 presents the proposed structure of developed DSS using TOPSIS/B-TOPSIS/MTOPSIS methodologies. An illustrative example are considered for the AGV selection with results and validation in Section 4. Finally, conclusion and future studies are provided in Section 5.

\section{PROPOSED MADM METHODOLOGY}

The proposed MADM methodology is divided in to various steps as described below:

Step 1: Identify the goal; find out all possible alternatives, selection attribute and its measures for the given application. An MADM problem with finite possibilities can be concisely expressed in matrix format as shown in Table 1 . In this Table, $A_{1}$, $A_{2}, \ldots, A_{m}$ are possible alternatives among which decision makers have to choose best alternative, $C_{1}, C_{2}, \ldots, C_{n}$ are attribute with which alternative performance are measured, $x_{i j}$ is the rating of alternative $i$ with respect to attribute $j, i=1,2 \ldots, m$; $\mathrm{j}=1,2 \ldots, \mathrm{n}$;

Step 2: The process of transforming attributes value into a range of $0-1$ is called normalization and it is required in MADM to transform performance rating with different data measurement unit in a decision matrix into a compatible unit. The attributes are of two types, beneficial (i.e. higher values are desired) and non-beneficial (i.e. lower values are desired). The values associated with the attributes $x_{i j}$ may be in different units (e.g., AGV cost expressed in dollars, Load capacity expressed in $\mathrm{Kg}$, etc.). The values of attributes can be normalized using the following equation: The normalized decision matrix is constructed using Eq.(1).

$$
r_{i j}=\frac{x_{i j}}{\sqrt{\sum_{j=1}^{n} x_{i j}^{2}}}
$$

where $r_{i j}$ is the normalized value obtained from decision matrix. It may be added here that Eq.(1) can deal with quantitative attributes. However, there exists some difficulty in the case of qualitative attributes (i.e. quantitative value is not available). A ranked value judgment on a fuzzy conversion scale is proposed in this paper by using fuzzy set theory. This approach is based on the work of [10]. The presented numerical approximation system systematically converts linguistic terms to their corresponding fuzzy numbers. An 11-point scale is proposed in this paper for better understanding and representation of the qualitative attribute. Table 2 represents the selection attribute on a qualitative scale using fuzzy logic, corresponding to the fuzzy conversion

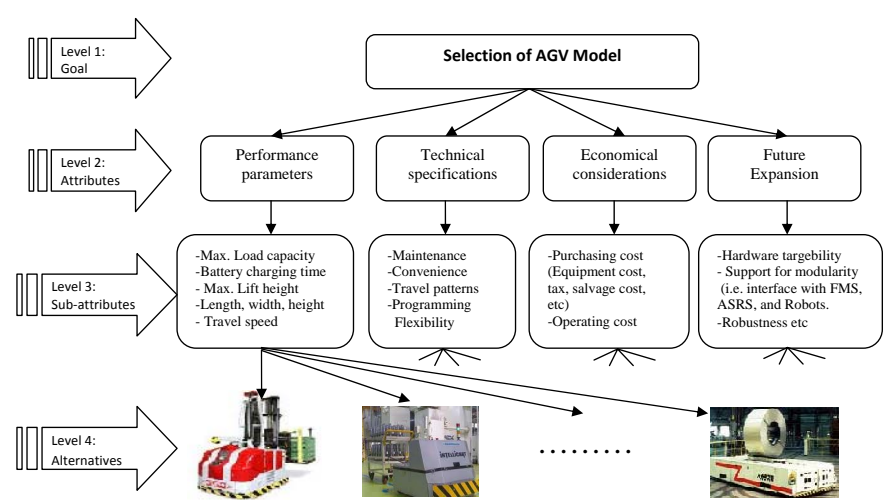

Fig. 1. Hierarchical structure of selecting AGV in AGVSEL system.

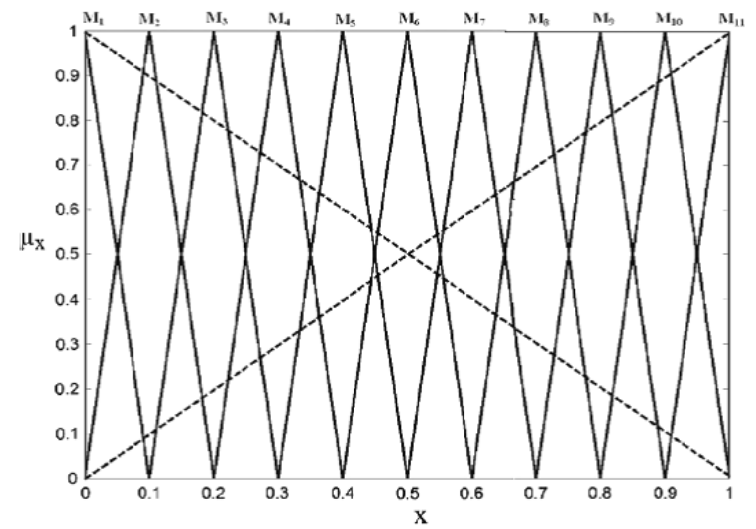

Fig. 2. Linguistic terms to fuzzy numbers conversion (11-point scale).

scale as shown in Fig.2. The list of possible fuzzy numbers that can be assigned in weighting the attributes and their linguistic correspondences are provided in Table 2 .

Step 3: Determination of weights of importance of the attributes In the following steps the subjective and objective weight computation procedure incorporated in AGVSEL system is proposed.

Step 3.1: Subjective weights of importance of the attributes The steps for the calculation of subjective weights $\left(W_{s}\right)$ are enumerated below.

First compute the relative importance of different attributes with respect to the objective. To do so, one has to construct a pairwise comparison matrix using a scale of relative importance. The judgments are entered using the fundamental scale of the analytic hierarchy process(AHP) as given in Table $3[11,12]$. An attribute compared with itself is always assigned the value 1 so the main diagonal entries of the pair-wise comparison matrix are all 1 . The numbers $3,5,7$, and 9 correspond to the verbal judgments moderate importance, strong importance, very strong importance, and absolute importance (with 2, 4, 6, and 8 for compromise between the previous values).

Next, the relative normalized weight $\left(W_{j}\right)$ of each attribute by (i) calculating the geometric mean of ith row and (ii) normalizing the geometric means of rows in the comparison matrix. This can be represented as

$$
G M_{i}=\left\{\prod_{j=1}^{n} a_{i j}\right\}^{1 / n}
$$


Table 1. A typical multiple attribute decision problem.

\begin{tabular}{|l|c|c|c|c|}
\hline Alternatives & Attribute $1\left(C_{1}\right)$ & Attribute $2\left(C_{2}\right)$ & $\ldots$ & Attribute $\mathrm{n}\left(C_{n}\right)$ \\
\hline$A_{1}$ & $x_{11}$ & $x_{12}$ & $\ldots$ & $x_{1 n}$ \\
\hline$A_{2}$ & $x_{21}$ & $x_{22}$ & $\ldots$ & $x_{2 n}$ \\
\hline$\cdot$ & $\cdot$ & $\cdot$ & $\ldots$ &. \\
\hline$A_{m}$ & $\cdot$ & $\cdot$ & $\ldots$ &. \\
\hline
\end{tabular}

Table 2. Linguistic terms of attribute weights and its values

\begin{tabular}{|l|c|c|}
\hline Linguistic terms & $\begin{array}{c}\text { Fuzzy } \\
\text { number }\end{array}$ & $\begin{array}{c}\text { Assigned } \\
\text { crisp score }\end{array}$ \\
\hline Exceptionally low & $M_{1}$ & 0.0455 \\
\hline Extremely low & $M_{2}$ & 0.1364 \\
\hline Very low & $M_{3}$ & 0.2273 \\
\hline Low & $M_{4}$ & 0.3182 \\
\hline Below average & $M_{5}$ & 0.4091 \\
\hline Average & $M_{6}$ & 0.5000 \\
\hline Above average & $M_{7}$ & 0.5909 \\
\hline High & $M_{8}$ & 0.6818 \\
\hline Very high & $M_{9}$ & 0.7727 \\
\hline Extremely high & $M_{10}$ & 0.8636 \\
\hline Exceptionally high & $M_{11}$ & 0.9545 \\
\hline
\end{tabular}

$$
W_{s j}=\frac{G M_{i}}{\sum_{i=1}^{n}\left(G M_{i}\right)}
$$

The geometric mean method of AHP is used in the present work to find out the relative normalized weights of the attributes because of its simplicity and easiness to find out the maximum Eigen value and to reduce the inconsistency in judgments.

Calculate matrix A3 and A4 such that A $3=A_{n x n}$ X A2 and A4 $=\mathrm{A} 3 / \mathrm{A} 2$, where $\mathrm{A} 2=W_{s j}=\left[W_{1}, W_{2}, \ldots W_{n}\right] \mathrm{T}$.

Find out the maximum Eigen value $\lambda_{\max }$ that is the average of matrix A4.

Consistency Test: Calculate the consistency index $\mathrm{CI}=\left(\lambda_{\max }-\right.$ $N) /(N-1)$. The smaller the value of CI, the smaller is the deviation from the consistency. Obtain the random index (RI) for the number of attributes used in decision making. The reference RI values for different numbers of $\mathrm{N}$ are shown in Table 4.

Calculate the consistency ratio: $\mathrm{CR}=\mathrm{CI} / \mathrm{RI}$. A value of $\mathrm{CR}$ of 0.1 or less is considered as acceptable and decision is taken for preparation of the judgment matrix otherwise the necessary changes are made while preparation of the judgment matrix.

Step 3.2: Objective weight calculation procedure

Shannon's entropy is a well known method for obtaining the objective weights for a MADM problem especially when obtaining a suitable weight based on the preferences are not possible. The procedure of Shannon's entropy can be expressed in a series of steps for the calculation of objective weights $\left(W_{o}\right)$ as per following steps given in [13].

First the normalized decision matrix is constructed using Eq.(1). After normalizing the decision matrix, the entropy values $e_{j}$ are calculated using Eq.(4).

$$
e_{j}=-k \sum_{j=1}^{n} r_{i j} l n r_{i j}
$$

where $\mathrm{k}$ is a constant, $\mathrm{k}=(\ln (n))^{-1}(\mathrm{n}$ indicates number of decision alternatives). The degree of divergence $d_{j}$ of the intrinsic information of each attrubute $(j=1,2, \ldots, n)$ may be calculated as

$$
d_{j}=1-e_{j}
$$

The value $d_{j}$ represents the inherent contrast intensity of attribute $\mathrm{j}$. The higher the $d_{j}$ is, the more important the attribute $\mathrm{j}$ is for the problem.

The objective weight $\left(W_{o}\right)$ for each criterion can be obtained by Eqs.(6).

$$
W_{o j}=\frac{d_{j}}{\sqrt{\sum_{k=1}^{n} d_{k}}}
$$

Step 3.3: Composite weights of importance of the attributes The weights obtained by subjective and objective methods are different and so also the ranking. If the decision maker models seamlessly subjectivity of DM's expertise and objectivity of attribute data then decision maker may use the composite weights described by the equation (7). The equation gives combination of subjective and objective weight spectrum.

$$
W_{c j}=\left[\beta W_{s j}+(1-\beta) W_{o j}\right]
$$

where $W_{c j}$ is the composite weight of jth attribute and $W_{s j}$ and $W_{o j}$ are the subjective and objective weights. $\beta$ is composite weight coefficient and the values are between 0 and 1 with an interval of 0.1. For subjective approach, S-100\%, $\beta=1 ; W_{c j}=$ $W_{s j}$, For objective approach O-100\%, $\beta=0 ; W_{c j}=W_{o j}$, and $\sum_{c=1}^{n} \mathrm{~W}_{o j}=1 ; 0 \leq \beta \leq 1$.

Step 4: Computation of AGV selection index (ASI)

TOPSIS/B-TOPSIS/M-TOPSIS methods are used for computation of ASI. TOPSIS one of the classical MADM methods was developed by Hwang and Yoon [10], B-TOPSIS by Shanian and Savadogo [14] and M-TOPSIS advocated by $[15,16]$ are used to get ASI to rank AGVs using crisp/fuzzy MADM approaches.

ASI by TOPSIS method. The reasons for choosing TOPSIS are that (1) the TOPSIS approach is rational and easy to comprehend, (2) the computations involved are simple. The steps of the fuzzy TOPSIS approach are presented by [17].

ASI by B-TOPSIS method. The block(B) TOPSIS method is a simplified version of TOPSIS, the two euclidean distances ideal and negative ideal solutions for each alternative are, calculated as: The distance of a alternative $j$ to the positive ideal solution $\left(D_{i}^{+}\right)$, and from the negative ideal solution $\left(D_{i}^{-}\right)$are calculated using Eqs. (2) and (3) given below by [14].

$$
D_{i}^{+}=\sum_{j=1}^{m}\left|v_{i j}-v_{j}^{+}\right| ; i=1,2, \ldots, n ; j=1,2, \ldots, m ;
$$


Table 3. Relative importance of attributes (aij)

\begin{tabular}{|l|l|}
\hline Relative importance (aij) & Description \\
\hline 1 & Attributes $\mathrm{i}$ and $\mathrm{j}$ are equally important \\
\hline 3 & Attribute $\mathrm{i}$ is moderately more important than attribute $\mathrm{j}$ \\
\hline 5 & Attribute $\mathrm{i}$ is strongly more important than attribute $\mathrm{j}$ \\
\hline 7 & Attribute $\mathrm{i}$ is very strongly more important than attribute $\mathrm{j}$ \\
\hline 9 & Attribute $\mathrm{i}$ is absolutely more important than attribute $\mathrm{j}$ \\
\hline $2,4,6,8$ & Intermediate values of relative importance \\
\hline
\end{tabular}

Table 4. Random Index (RI) values for corresponding matrix size (Saaty 2004).

\begin{tabular}{|l|c|c|c|c|c|c|c|}
\hline Attributes (n) & 1 & 2 & 3 & 4 & 5 & 6 & 8 \\
\hline RI values & 0 & 0 & 0.52 & 0.89 & 1.11 & 1.25 & 1.35 \\
\hline
\end{tabular}

$$
D_{i}^{-}=\sum_{j=1}^{m}\left|v_{i j}-v_{j}^{-}\right| ; i=1,2, \ldots, n ; j=1,2, \ldots, m
$$

Where $v_{i j}$ are weighted normalized values and $v_{j}^{+}, v_{j}^{-}$are the positive ideal and negative ideal solutions.

ASI by M-TOPSIS method. M-TOPSIS is a method sourced from TOPSIS. Of the two methods which are discussed above, we believe that the M-TOPSIS is more reasonable because the ranking order of alternatives was determined by their distances to optimized ideal reference point. Also, equation to calculate ASI of M-TOPSIS is more accurate than equation of TOPSIS/BTOPSIS. It can solve the problems of TOPSIS/B-TOPSIS such as rank reversals and evaluation failure when alternatives are symmetrical [17].

Step 5: Ranking and final selection of AGV. A final decision is taken keeping in view of the attribute data supplied by the industry. All possible constraints likely to be experienced by the DM are looked in during this stage. These include constraints such as maximum load to be carried, maximum lift height, maximum Travel speed, budgetary provision. However, compromise may be made in favor of an alternative with a higher value of ASI. In the next section, we present the structure of the decision support system - AGVSEL.

\section{STRUCTURE OF THE DECISION SUPPORT SYSTEM - AGVSEL}

In the development of AGVSEL, the attributes have been hierarchically structured in four levels (see, Fig. 1), in accordance with the MADM framework. The level 1 contains the overall objective for AGV selection. Level 2 consists of four groups of attributes. Level 3 contains the detailed attributes under each of the above groups. Level 4 contains the list of AGVs available in market. The developed decision support system incorporates five separate modules (see, Fig. 3), namely, database module, preranking module, weight determination module, ranking module and analysis module. Pre-ranking module prompts the user to answer 16 questions to eliminate the non confirming AGVs and obtain a feasible set of AGVs. The feasible sets of AGVs are then ranked by TOPSIS/B-TOPSIS and M-TOPSIS methods. The entire system has been developed in a software program using Visual Basic in Windows environment. Details of the modules are described in the following sections.

\subsection{Database module}

This module comprises of AGV database of 114 AGVs from 76 manufacturers worldwide. One can add or delete a new AGV in the AGVSEL system database. In developed system, the database includes different attributes (see, Fig. 1). There was no information available about attribute data in published literature for AGV selection. However, to fill this gap the data was taken from online mode, viz.,the HK Systems (http://www.hksystems.com),

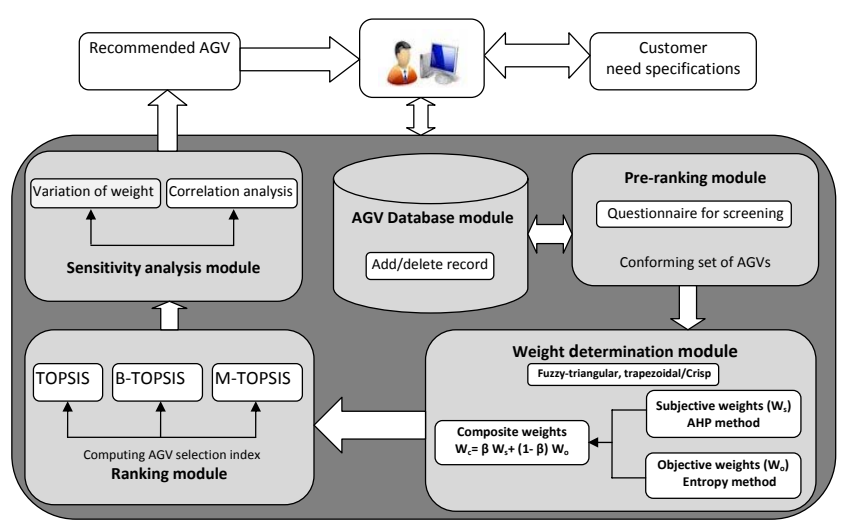

Fig. 3. The Structure of AGVSEL system.

Egemin automation (http://www.egemin.com), Corecon automation (http://www.coreconagvs.com), Amerden Inc (http://www.amerden.com), Savant automation, (http://www.agvsystems.com), JBT corporation (http://www.jbtcagv.com) and Jervis B Webb Company (http://www.jervisbwebb.com), etc which are the key players in the AGV business. A feasible set of AGVs is screened from this extensive database using the pre-ranking module described in the next section.

\subsection{Pre-ranking module}

The pre-ranking module of the AGVSEL, (see, Fig.4) poses a questionnaire to the decision maker with answers. The user has the choice to answer suitable number of questions. The minimum and maximum values of load to be moved are entered in response to the first question. The transport unit size, i.e., length, width and height, transport units that will make up the goods to be transported, distance to be travelled, available floor-space, budget allocated, etc., are entered through questions 2 to 7 . The number of pick-up and drop-off points, maximum lift height, minimum turning radius, labor requirements are entered in questions 8 to 11 . Question 12 to 16 calculate the expansion in near future, battery charging details, reconfiguration time, any particular environmental conditions to be taken into account, or any other information user wants to provide can be entered in response to this questions. Once the broad specifications are entered by the decision maker in the pre-ranking module, the feasible set of AGVs are determined by this module and is provided on the console (see, Fig. 5). The feasible set can be simultaneously passed on to the weight determination procedure as discussed in section 2 step 3 for assigning weights.

\subsection{Ranking module}

Once the weights are entered, click the button labeled as TOPSIS/B-TOPSIS/M-TOPSIS results will provide the AGV selection index and the ranking of the AGVs. TOPSIS/B- 


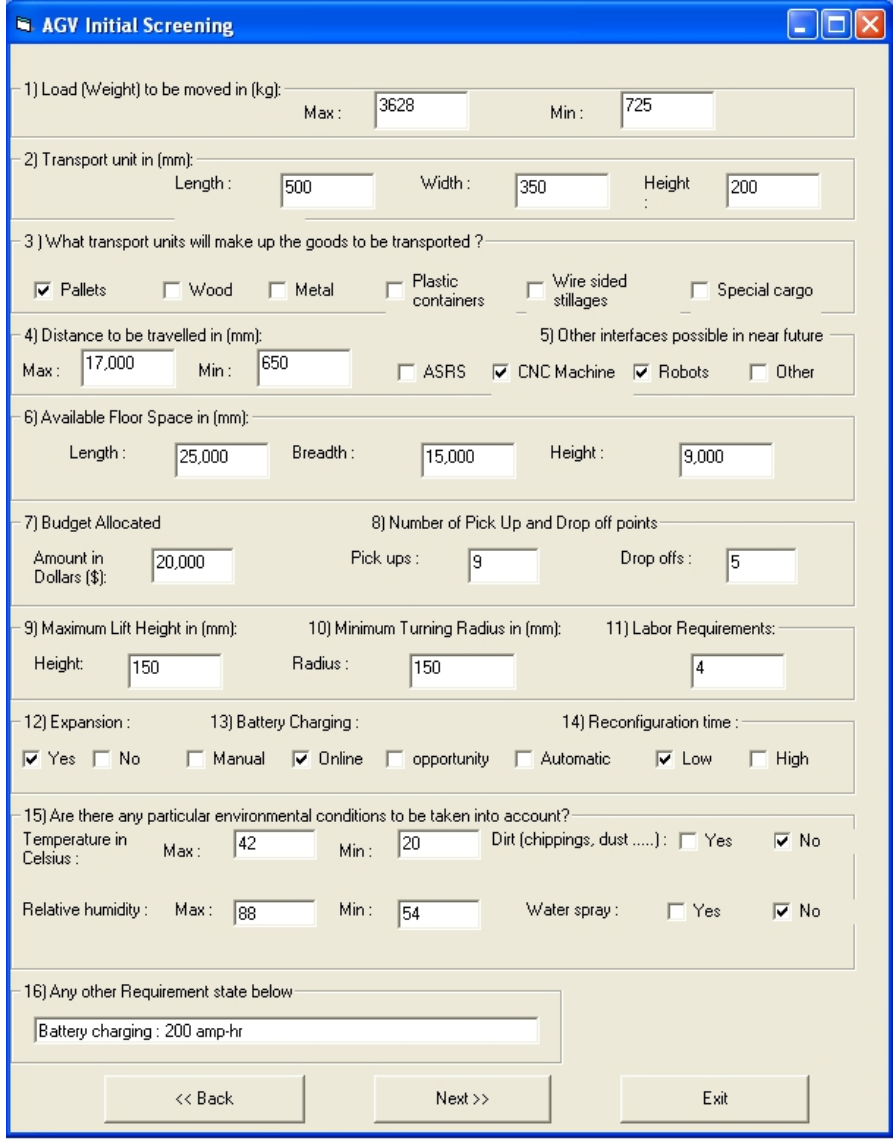

Fig. 4. Pre ranking module of AGVSEL.

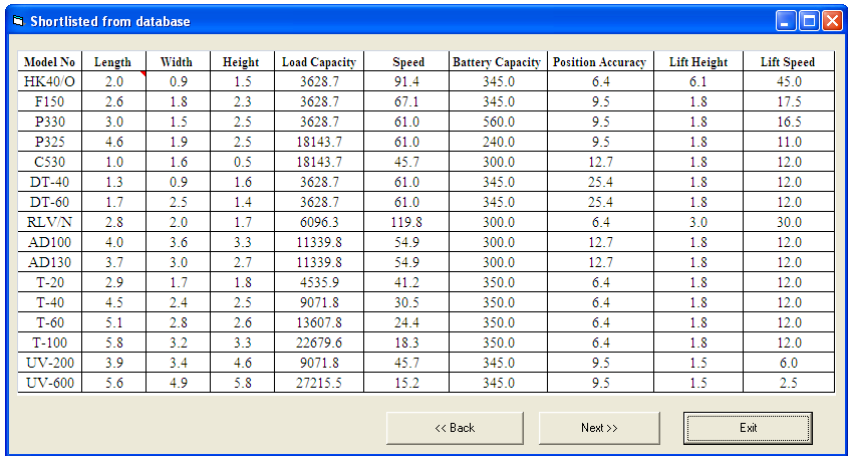

Fig. 5. output screen of AGVSEL system for the pre ranking module

TOPSIS/M-TOPSIS ranking methods are used for ranking feasible AGVs. In the following section the ranking methods used in AGVSEL system are briefly discussed.

3.3.1 AGV selection index by TOPSIS method. The reasons of choosing TOPSIS are that (1) the TOPSIS approach is rational and easy to comprehend, (2) the computations involved are simple, (3) the concept allows both subjective and objective weights to be aggregated in the decision-making process. The readers can referred to $[10,17]$ for detailed explanations and applicational steps of crisp/fuzzy TOPSIS approach.

3.3.2 AGV selection index by B-TOPSIS method. The block(B) TOPSIS method is a simplified version of TOPSIS, the two euclidean distances ideal and negative ideal solutions for each alternative are, calculated as: The distance of a alternative $j$ to the positive ideal solution $\left(D_{i}^{+}\right)$, and from the negative ideal solution $\left(D_{i}^{-}\right)$are calculated using Eqs. (2) and (3) given below by [14].

$$
\begin{aligned}
& D_{i}^{+}=\sum_{j=1}^{m}\left|v_{i j}-v_{j}^{+}\right| ; i=1,2, \ldots, n ; j=1,2, \ldots ., m ; \\
& D_{i}^{-}=\sum_{j=1}^{m}\left|v_{i j}-v_{j}^{-}\right| ; i=1,2, \ldots, n ; j=1,2, \ldots, m ;
\end{aligned}
$$

Where $v_{i j}$ are weighted normalized values and $v_{j}^{+}, v_{j}^{-}$are the positive ideal and negative ideal solutions.

3.3.3 AGV selection index by M-TOPSIS method. M-TOPSIS is a method sourced from TOPSIS. Of the two methods discussed above, we believe that the M-TOPSIS is more reasonable because the ranking order of alternatives was determined by their distances to optimized ideal reference point [16]. Also, equation to calculate index value of M-TOPSIS is more accurate than equation of TOPSIS/B-TOPSIS. It can solve the problems of TOPSIS/B-TOPSIS such as rank reversals and evaluation failure when alternatives are symmetrical. The ranking obtained with TOPSIS/B-TOPSIS methods are compared with M-TOPSIS for validation purpose and to check consistency in ranking.

\subsection{Analysis module}

In the analysis module, the user enters the subjective weights directly as crisp/fuzzy numbers (see, Fig. 6) and calculates the consistency ratio. The ratio value of 0.1 or less is considered as acceptable as it reflects an informed judgment that could be attributed to the knowledge of the DM about the problem under study. The rankings are then obtained for each set of weights using TOPSIS/B-TOPSIS/M-TOPSIS methods. An example of the output screen by using TOPSIS method is provided in Fig. 6. In the output screen, the feasible AGVs are listed according to their ranking scores. The user can see the ranking scores and technical specifications in the same screen. We can also obtain ranking of AGVs by clicking on nine different sets of combinations of subjective and objective weights (see, Fig. 6). The correlation analysis can also be carried out to test the accuracy of rankings obtained. In the next section, we present results for the AGV selection index calculations by considering industrial case. The results are presented for variation composite weights followed by a brief discussion.

\section{RESULTS AND DISCUSSIONS}

\subsection{AGV selection using quantitative data}

An AGV selection example using quantitative data for a manufacturing enterprise in Maharashtra, India is presented using the developed AGVSEL system.

Step 1: The requirements and attribute information of the AGVs as desired by the industry are listed in Table 5 . Of the nineteen attributes incorporated in AGVSEL, we have opted for ten attributes data on the basis of customer requirements. The maximum budgetary provision limits the number of AGVs to select from. Among remaining nine attributes, eight attributes, viz., MLC, MLH, MS, L,W,H, battery capacity are the beneficial attributes, i.e., Higher values are desired and purchasing cost (PC), $\mathrm{P}$ is non-beneficial attributes, i.e., Lower values are desired. Thus, attributes MS to P (see, Table 5) are not specified by the customer in quantitative terms, but included to improve the effectiveness of pre-ranking module by assigning desired degree, i.e., High or low. These specifications when applied to the AGV database incorporated in the system filter out 16 feasible AGVs from a total of 114 . 
Table 5. Information about the AGV from industry.

\begin{tabular}{|l|c|}
\hline Specifications & Values \\
\hline Maximum load to be carried (MLC): & $3600 \mathrm{~kg}(3.6$ tons $)$ \\
Maximum lift height (MLH): & $150 \mathrm{~mm}$ \\
Battery should not get discharge before : & $4 \mathrm{hrs}$ \\
Maximum budgetary provision: & $\$ 20,000$ \\
Maximum Travel speed (MS): & High \\
Length of AGV (L): & High \\
Width of AGV (W): & High \\
Height of AGV (H): & High \\
Purchasing cost (PC): & Low \\
Position accuracy (P): & High \\
\hline
\end{tabular}

Table 6. Comparision of the attribute weights for AGV selection.

\begin{tabular}{|l|c|c|c|c|c|c|}
\hline \begin{tabular}{l|l|c|c|} 
Methods \\
Attributes
\end{tabular} & SW & OW & CW & IW & CombineW \\
{$[1,12]$} & {$[13]$} & 0.059 & 0.042 & 0.081 \\
L & 0.072 & 0.092 & 0.056 & 0.045 & 0.079 \\
W & 0.072 & 0.087 & 0.073 & 0.035 & 0.090 \\
H & 0.072 & 0.113 & 0.325 & 0.043 & 0.191 \\
MLC & 0.170 & 0.214 & 0.090 & 0.046 & 0.100 \\
MS & 0.092 & 0.109 & 0.022 & 0.619 & 0.090 \\
B & 0.170 & 0.015 & 0.158 & 0.070 & 0.133 \\
P & 0.151 & 0.117 & 0.079 & 0.067 & 0.092 \\
LH & 0.104 & 0.085 & 0.134 & 0.031 & 0.134 \\
LS & 0.092 & 0.163 & 0.095 & 0.123 \\
\hline SW-Subjective weights, OW-Objective weights, CW-Compromised weighting method, IW-Integrated weight method, CombineW-Combined weight method, PW-proposed
\end{tabular}
weight method.

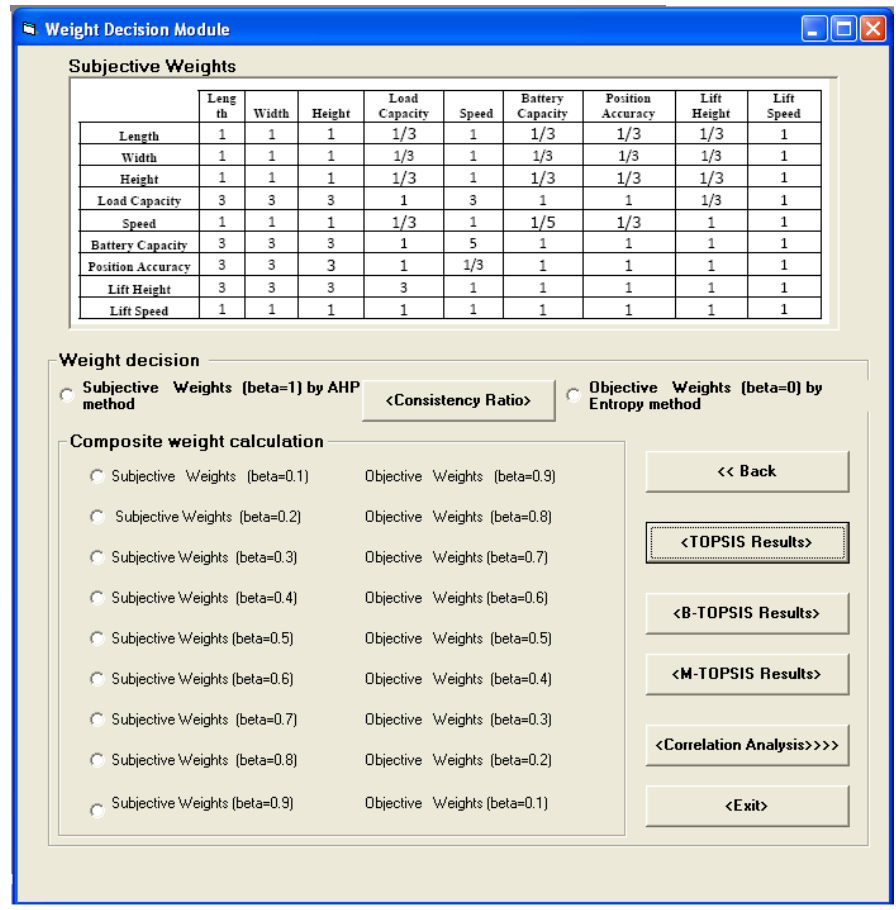

Fig. 6. Input screen for the weights in the weight decision module.

Step 2: The values of the attributes are normalized using Eq. (1) and are not shown here due to space constraint.

Step 3: Next, subjective weights (by AHP method) explained in step 3.1 are calculated with the DM's expertise (see, Table 6). The consistency ratio of the pairwise comparison matrix is calculated to be 0.0487 . The ratio value of 0.1 or less is considered as acceptable as it reflects an informed judgment of the DM and weights obtained are used in the selection process. The objective weights are calculated by using entropy method as explained in step 3.2. The composite weights of importance of the attributes

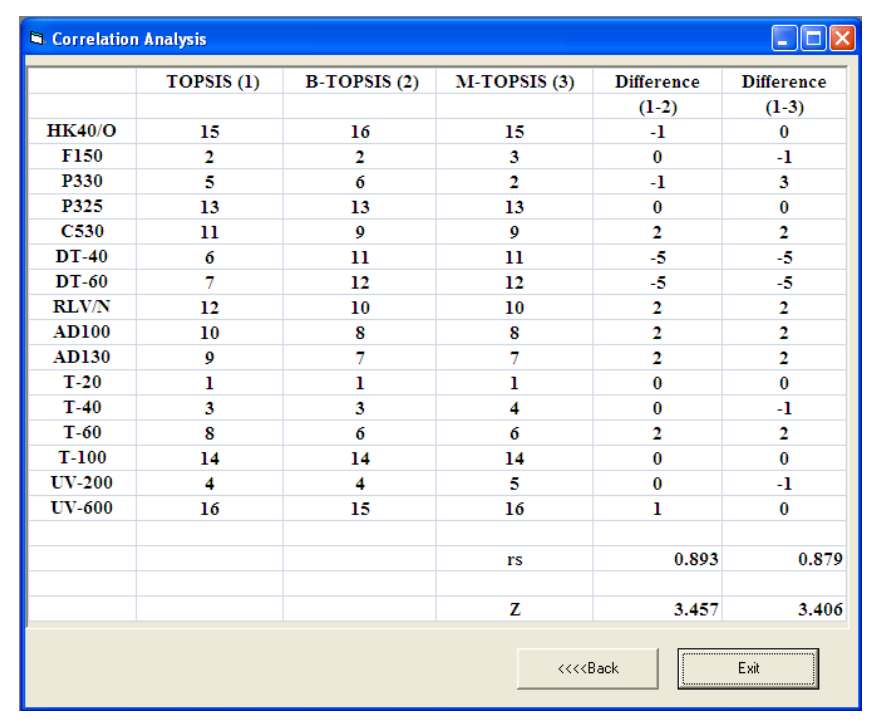

Fig. 7. output screen for the spearmans rank correlation test.

are computed using Eq.(8) for different composite weight coefficient $(\beta)$ and the values are between 0 and 1 with an interval of 0.1 . It may be mentioned here that for $\beta=1$, the composite weights are same as that of subjective weights of step 3.1 and for and $\beta=0$, the composite weights are same as that of objective weights of step 3.2. We found that in case of subjective weights i.e. $\beta=1$, the attributes MLC, $\mathrm{L}, \mathrm{W}$ and battery capacity have higher weight values compared to other attributes. So also, the attributes H, LS has higher weight values compared to MS, P, LH. It is known from MADM theory that, the attributes having lower weights have no major effect on the final selection.

Step 4: Computation of AGV selection index (ASI) and the ranking obtained are presented using the TOPSIS/B-TOPSIS/MTOPSIS methods for subjective, objective and composite weights in Table 7. The AGVs are arranged in the descending order of their selection index for TOPSIS/B-TOPSIS method and 
Table 7. AGV selection index, $\operatorname{rank}(\mathrm{R})$ using TOPSIS(T), B-TOPSIS(B-T), M-TOPSIS(M-T) for subjective(SW) and objective weights $(\mathrm{OW})$.

\begin{tabular}{|c|c|c|c|c|c|c|c|c|c|c|c|c|}
\hline \multirow[t]{2}{*}{ Alternatives } & \multicolumn{5}{|c|}{ SW } & \multicolumn{7}{|c|}{ OW } \\
\hline & $\mathrm{T}$ & $\mathrm{R}$ & B-T & $\mathrm{R}$ & M-T & $\mathrm{R}$ & $\mathrm{T}$ & $\mathrm{R}$ & $\mathrm{B}-\mathrm{T}$ & $\mathrm{R}$ & M-T & $\mathrm{R}$ \\
\hline $\mathrm{HK} 40 / \mathrm{O}$ & 0.573 & 15 & 0.407 & 16 & 0.059 & 15 & 0.524 & 15 & 0.392 & 16 & 0.085 & 15 \\
\hline $\mathrm{F} 150$ & 0.768 & 2 & 0.768 & 2 & 0.013 & 3 & 0.743 & 4 & 0.743 & 4 & 0.019 & 3 \\
\hline P330 & 0.698 & 5 & 0.698 & 6 & 0.027 & 2 & 0.752 & 2 & 0.752 & 2 & 0.017 & 2 \\
\hline P325 & 0.636 & 13 & 0.636 & 13 & 0.041 & 13 & 0.605 & 12 & 0.605 & 12 & 0.055 & 12 \\
\hline C530 & 0.650 & 11 & 0.650 & 9 & 0.038 & 9 & 0.647 & 11 & 0.647 & 11 & 0.044 & 11 \\
\hline DT-40 & 0.645 & 6 & 0.645 & 11 & 0.040 & 11 & 0.724 & 5 & 0.724 & 5 & 0.024 & 5 \\
\hline DT-60 & 0.638 & 7 & 0.638 & 12 & 0.041 & 12 & 0.717 & 6 & 0.717 & 6 & 0.026 & 6 \\
\hline RLV/N & 0.649 & 12 & 0.649 & 10 & 0.038 & 10 & 0.589 & 13 & 0.589 & 13 & 0.060 & 13 \\
\hline AD100 & 0.677 & 10 & 0.677 & 8 & 0.034 & 8 & 0.663 & 10 & 0.663 & 10 & 0.041 & 10 \\
\hline AD130 & 0.694 & 9 & 0.694 & 7 & 0.031 & 7 & 0.684 & 8 & 0.684 & 8 & 0.037 & 9 \\
\hline $\mathrm{T}-20$ & 0.832 & 1 & 0.832 & 1 & 0.000 & 1 & 0.824 & 1 & 0.824 & 1 & 0.000 & 1 \\
\hline T-40 & 0.765 & 3 & 0.765 & 3 & 0.014 & 3 & 0.746 & 3 & 0.746 & 3 & 0.020 & 4 \\
\hline T-60 & 0.701 & 8 & 0.701 & 5 & 0.027 & 6 & 0.677 & 9 & 0.677 & 9 & 0.037 & 8 \\
\hline $\mathrm{T}-100$ & 0.578 & 14 & 0.578 & 14 & 0.055 & 14 & 0.545 & 14 & 0.545 & 14 & 0.072 & 14 \\
\hline UV-200 & 0.720 & 4 & 0.719 & 4 & 0.023 & 5 & 0.702 & 7 & 0.702 & 7 & 0.029 & 7 \\
\hline UV-600 & 0.519 & 16 & 0.519 & 15 & 0.073 & 16 & 0.505 & 16 & 0.505 & 15 & 0.093 & 16 \\
\hline
\end{tabular}

Table 8. Rankings of the AGV models by different weight generation methods.

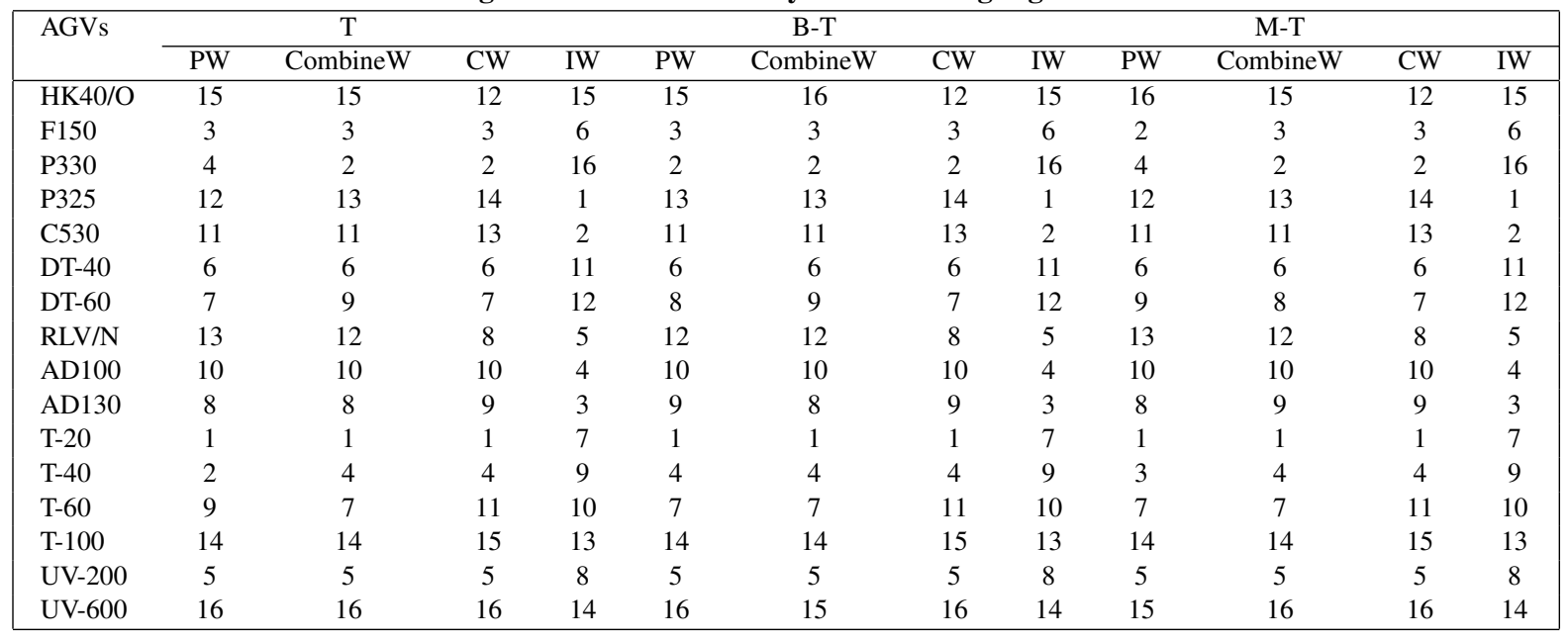

ascending order of their selection index for M-TOPSIS method. It can be seen that the match between the TOPSIS, B-TOPSIS and M-TOPSIS method is very good and is confirmed by Spearman's test.

Step 5: The obtained ranking is discussed with the customer so as to ascertain the suitability amongst the top three alternatives. It was found that these alternatives conform with the desired specifications to a great extent. The AGV model T-20 can be considered the best choice as determined by TOPSIS, B-TOPSIS, and M-TOPSIS methods respectively and AGV UV600 is the last choice of the decision maker for a given application.

\subsection{Effect of composite weights}

Next, the effect of proposed composite weights on final ranking is discussed here. The top ranked AGV using TOPSIS method is $\mathrm{T}-20$ for $\beta$ ranging from 0 to 1.0 at an interval of 0.2 . Rank transition is observed for second position between P-330 to T40 with the variation in $\beta$ (see, Table 8 ), in case of B-TOPSIS method T-20 remains at top but second position is occupied by $\mathrm{P}-330$ for $\beta$ ranging from 0 to 0.2 , T40 for $\beta$ ranging from 0.3 to 0.4 and F-150 for $\beta$ ranging from 0.4 to 1.0 , followed by T40, F-150 for third rank (see, Table 8). T-20 remains at top if we rank feasible AGVs using M-TOPSIS method second position is occupied by P-330 and third position by F-150 (see, Table 8). In general, it does not matter that the different methods give different rankings, so long as the first choice AGV is consistent. The results obtained provide valuable insight into the rank transition taking place with the variation in composite weight caused by $\beta$. The results are indicative of the fact that selection of the best AGV from the feasible set not only depends on ranking method but also on the interpolating weight factor $\beta$. In this illustration, top three AGV are T-20, P-330 and T-40 are the best choices and don't need further scrutiny with respect to their specifications and these AGVs can be selected for the given application. Thus, this system helps DM's to visualize the changes in the ranking of AGV with $\beta$. The system quickly and efficiently simulates "“what if?" scenario for different composite weights and helps in developing insight into the complex interplay of composite weights. The AGVSEL system can be generalised so that it can consider any number of quantitative and qualitative fuzzy AGV selection attributes. Further, the it can be used for any other type of decision making situations as well with minor modifications in software code.

\subsection{Validation of the proposed method}

In this section, our proposed composite weight approach is compared and validated with the compromised weighting $(\mathrm{CW})$ method [18],integrated weight (IW) method [19], combined weight (CombineW) method [20] to the AGV selection problem and provide some numerical results. The weights of the nine attributes determined by the above four methods (see, Table 6) and the rankings of the $16 \mathrm{AGV}$ models under the four differ- 
ent sets of attribute weights are shown in Table 8. We found that the weights of attributes obtained by the four methods are different, particularly combined weight method and proposed composite weight method give similar weights. So also the rankings of the $16 \mathrm{AGV}$ alternatives derived by the combined weighting and proposed composite weight model are very close but,they are not similar in case of compromised weighting method and integrated weight method. While it is natural that different approaches will usually lead to different results in a multiple attribute decision making problem and the adoption of a specific approach is up to the decision maker's preference and the decision environment. Through the above examination and analyses, it can be summarized that the proposed weight method has the following advantages: The weights of attributes determined using the proposed method are more comprehensive and convincing as these are obtained by interpolating subjective and objective weights. The proposed method not only incorporates the amount of information each attribute contains but, also DM's subjective preferences while calculating weights. The rank transition taking place between different alternatives is visible and this effect can be modelled with proposed composite weight model.

\subsection{Consistency in ranking using Spearman's test}

The statistical significance of the difference between the ranking obtained for the TOPSIS approach and for the BTOPSIS/M-TOPSIS approach is determined using Spearman's rank-correlation test. This test is a special form of correlation test used when the actual values of paired data are substituted with the ranks which the values occupy in the respective samples. In this study, Spearman's test evaluates the similarity of the rankings of the AGVs using TOPSIS and B-TOPSIS/M-TOPSIS methods. In the application of Spearman's test, to test the null hypothesis $\left(H_{0}\right.$ : There is no similarity between the two rankings), a test statistic, Z, is calculated using Eqs. (12) and (13) and compared with a pre-determined level of significance value.

$$
\begin{gathered}
r_{s}=1-\frac{6 \sum_{j=1}^{k}\left(d_{j}\right)^{2}}{K\left(K^{2}-1\right)} \\
Z=r_{s} \sqrt{K-1}
\end{gathered}
$$

In this study, 1.645, which corresponds to the critical $\mathrm{Z}$ value at the level of significance of $\alpha=0.05$, is selected. If the test statistic computed by Eq. (13) exceeds 1.645, the null hypothesis is rejected and it is to be concluded that $H_{1}$ : The two rankings are similar is true. In Eqs. (12) and (13), $d_{j}$ is the ranking difference of alternatives $j$, and $k$ is the number of alternatives to be compared. $r_{s}$ represents the Spearman's rank correlation coefficient in Eqs. (12) and (13). The rankings obtained by the TOPSIS, BTOPSIS and M-TOPSIS in the AGV selection problem are provided in Fig. 6. The Spearman rank correlation coefficient between the methods is 0.8930 and 0.8790 which shows high correlation between the rankings proposed by the two methods. The comparison is performed by taking the difference of the ranks of the TOPSIS, B-TOPSIS and M-TOPSIS, and then calculating Spearman's correlation coefficient (Z-value) for the difference. The calculated Z-value, 3.850, is higher than 1.645 , which implies that the difference in the two ranking results is statistically insignificant. Based on the test results, it is concluded that the rankings obtained by the test are realistic.

\section{CONCLUSIONS}

The composite weight based MADM method presented in this paper is incorporated in the decision support system (AGVSEL). It provides the decision maker a great deal of flexibility to combine subjective and objective weights in terms of weighting fac- tor $\beta$ and arrive at rank. The effectiveness of the method is examined with an illustrative example of selection of AGV for an industrial application. The incorporation of decision makers knowledge and database of most AGV models available in market into the support system has made the AGVSEL more comprehensive. The system quickly and efficiently simulates " what if?" scenario for different composite weights and helps in developing insight into the complex interplay of subjective and objective weights. In example, the AGV model T-20 can be considered as the best choice as determined by TOPSIS, B-TOPSIS, and MTOPSIS methods along spectrum of weights and AGV UV-600 is the last choice of the decision maker for a given application. The results obtained provide valuable insight into the rank transition taking place at intermediate position with the variation of $\beta$. The Spearman rank correlation coefficient between the methods is 0.8930 and 0.8790 which shows high correlation between the rankings proposed by the methods. Validation of the proposed method at $(\beta=0.5)$ with the combined weight method [20], compromised weighting method [18] and integrated weight method [19] shows T-20 as best choice and UV-600 as the last choice. Sensitivity analysis reveals that at moderate value of interpolating factor $(\beta)$ rank transition takes place for topmost position thereby achieving better insight into the complex interplay of subjective and objective weights.

\section{REFERENCES}

[1] Lothar S., Sebastian B., \& Stefan B., Automated Guided Vehicle Systems: a Driver for Increased Business Performance In Proceedings of the International MultiConference of Engineers and Computer Scientists 2008 Vol II IMECS 2008.

[2] Chan F.T.S., Ip R.W.L., \& Lau H., Integration of expert system with analytic hierarchy process for the design of material handling equipment selection system, Journal of Materials Processing Technology, (2001) 116:137-145.

[3] Fonseca D.J., Uppal G.,\& Greene T.J., A knowledge-based system for conveyor equipment selection., Expert Systems with Applications, (2004) 26:615-623.

[4] Kulak O., A decision support system for fuzzy multiattribute selection of material handling equipments., Expert Systems with Applications, (2005) 29:310-319.

[5] Chakraborthy S., \& Banik D., Design of a material handling equipment selection model using analytic hierarchy process. The International Journal of Advanced Manufacturing Technology, (2006) 28:1237-1245.

[6] Dağdeviren M., Decision making in equipment selection: an integrated approach with AHP and PROMETHEE. Journal of Intelligent Manufacturing, (2008) 19:397-406.

[7] $\dot{I} C ̧$, Yusuf Tansel, \& Yurdakul, M. Development of a decision support system for machining center selection., Expert Systems with Applications, (2009) 36:3505-3513.

[8] Tuzkaya G., Gúlsun B., Kahraman C., \& Ozgen D., An integrated fuzzy multi-criteria decision making methodology for material handling equipment selection problem and an application. Expert Systems with Applications, (2010) 37:2853-2863.

[9] Maniyaa K. D., and Bhatt M. G., A multi-attribute selection of automated guided vehicle using the AHP/M-GRA technique. International Journal of Production Research, (2011) 49: 6107-6124.

[10] Hwang C.L., Yoon K., Multiple Attribute Decision Making Methods and Applications, Springer, Berlin, Heidelberg, 1991.

[11] Saaty, T.L., The Analytic Hierarchy Process, McGraw Hill, New York, 1980. 
[12] Saaty, T.L., How to make a decision: the analytic hierarchy process, Interfaces ,(1994) 24(6):1943.

[13] Shannon, C.E., \& Weaver W., The Mechanical Theory of Communication., University of Illions Press, 1947.

[14] Shanian A., \& Savadogo O., TOPSIS multiple-criteria decision support analysis for material selection of metallic bipolar plates for polymer electrolyte fuel cell. Journal of Power Sources, (2006) 159: 1095-1104.

[15] Deng H., Yeh C.H.,\& Willis R.J., Inter-company comparison using modified TOPSIS with objective weights. Computers and Operations Research, (2000) 27: 963-973.

[16] Ren, L., Y. Zhang, Y.Wang \& Z. Sun., Comparative Analysis of a Novel M-TOPSIS Method and TOPSIS, Applied Mathematics Research eXpress, (2007) Vol. 7, Article ID abm005, 10 pages, doi:10.1093/amrx/abm005.
[17] İÇ, Yusuf Tansel, \& Yurdakul, M., Development of a quick credibility scoring decision support system using fuzzy TOPSIS. Expert Systems with Applications, (2010) 37:567-574.

[18] Feng, C., \& Chen, C., The determination of criteria weights compromised weighting method. Traffic and Transportation, (1992) 14: 51-67.

[19] Ustinovichius L., Zavadskas E.K. \& Podvezko V., Application of a quantitative multiple criteria decision making (MCDM-1) approach to the analysis of investments in construction. Control and Cybernetics, (2007) 36(1) 251-268.

[20] Kong F. \& Liu H., Applying fuzzy analytic hierarchy process to evaluate success factors of e-commerce. International Journal of Information and System Sciences, (2005) 1(3-4) 406-412. 